PREPARED FOR THE U.S. DEPARTMENT OF ENERGY, UNDER CONTRACT DE-AC02-76CH03073

PPPL-3547

PPPL-3547

UC-70

Radial Patterns of Instability and Transport in JT-60U Internal Transport Barrier Discharges

by

G. Rewoldt, K.W. Hill, R. Nazikian, W.M. Tang,

H. Shirai, Y. Sakamoto, Y. Kishimoto, S. Ide, and T. Fujita

February 2001

NM|

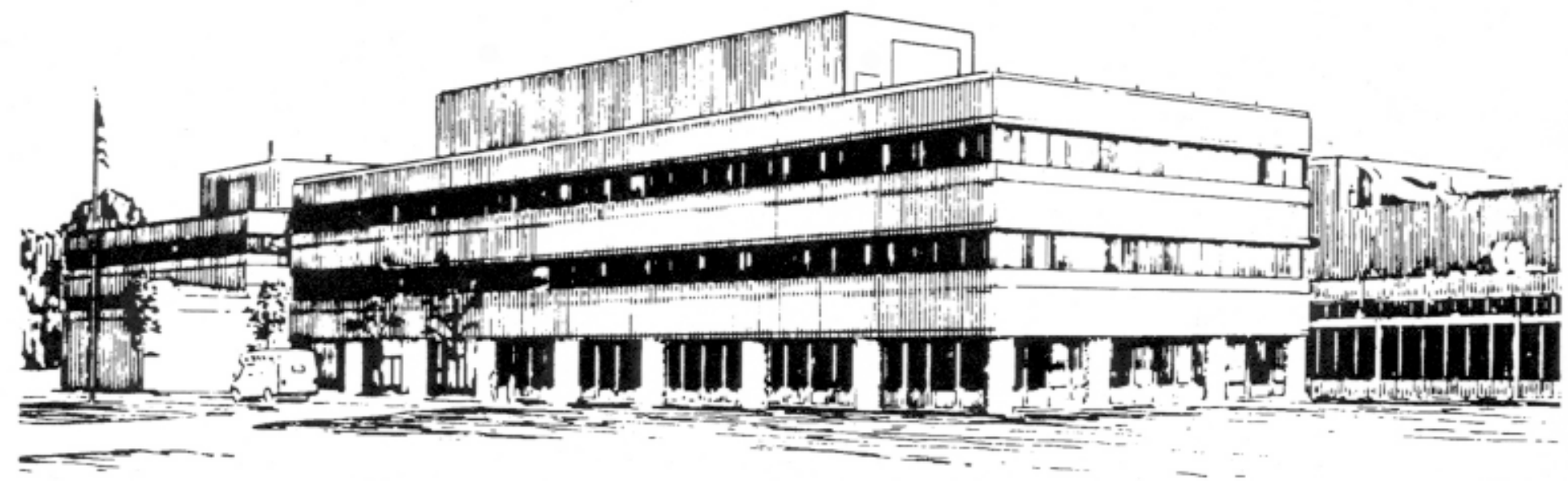

PRINCETON PLASMA PHYSICS LABORATORY PRINCETON UNIVERSITY, PRINCETON, NEW JERSEY 


\section{PPPL Reports Disclaimer}

This report was prepared as an account of work sponsored by an agency of the United States Government. Neither the United States Government nor any agency thereof, nor any of their employees, makes any warranty, express or implied, or assumes any legal liability or responsibility for the accuracy, completeness, or usefulness of any information, apparatus, product, or process disclosed, or represents that its use would not infringe privately owned rights. Reference herein to any specific commercial product, process, or service by trade name, trademark, manufacturer, or otherwise, does not necessarily constitute or imply its endorsement, recommendation, or favoring by the United States Government or any agency thereof. The views and opinions of authors expressed herein do not necessarily state or reflect those of the United States Government or any agency thereof.

\section{Availability}

This report is posted on the U.S. Department of Energy's Princeton Plasma Physics Laboratory Publications and Reports web site in Calendar Year 2001. The home page for PPPL Reports and Publications is: http://www.pppl.gov/pub_report/

DOE and DOE Contractors can obtain copies of this report from:

U.S. Department of Energy

Office of Scientific and Technical Information

DOE Technical Information Services (DTIS)

P.O. Box 62

Oak Ridge, TN 37831

Telephone: (865) 576-8401

Fax: (865) 576-5728

Email: reports@adonis.osti.gov

This report is available to the general public from:

National Technical Information Service

U.S. Department of Commerce

5285 Port Royal Road

Springfield, VA 22161

Telephone: 1-800-553-6847 or

(703) 605-6000

Fax: (703) 321-8547

Internet: http://www.ntis.gov/ordering.htm 


\title{
Radial Patterns of Instability and Transport in JT-60U Internal Transport Barrier Discharges
}

\author{
G. Rewoldt, K.W. Hill, R. Nazikian, W.M. Tang \\ Princeton Plasma Physics Laboratory, Princeton University \\ Princeton, New Jersey, United States of America \\ H. Shirai, Y. Sakamoto, Y. Kishimoto, S. Ide, T. Fujita \\ Japan Atomic Energy Research Institute \\ Mukouyama, Naka-machi, Naka-gun, Ibaraki-ken, Japan
}

\begin{abstract}
One class of internal transport barrier discharges in the JT-60U tokamak is characterized by two relatively flatter regions of the pressure separated by a region with very large pressure gradient. Linear growth rates for toroidal drifttype modes are calculated for discharges in this class, without and with sheared $\mathbf{E} \times \mathbf{B}$ rotation effects. For cases with fully-developed barriers, the results with rotation are consistent with a picture in which the radial electric field generated in part by the steep pressure gradient causes local stabilization, and thus reduction of the local anomalous transport, which allows the steep pressure gradient to persist. If rotation is omitted from the calculation for these cases, or if rotation is included for cases without barriers or with partially developed barriers, the unstable region spreads into the steep pressure gradient region.
\end{abstract}




\section{G. Rewoldt, et al.}

\section{Introduction}

One class of internal transport barrier (ITB) discharges in the JT-60U tokamak, that of the "box-type" ITBs, is characterized by radial profiles of the total pressure with relatively flat inner and outer regions separated by a relatively steep barrier region. However, for these sorts of pressure profiles to be able to persist, the transport in the steep barrier region has to be correspondingly small, though it can remain large in the inner and outer relatively flat regions. The anomalous transport that governs the evolution of the density and temperature, and therefore pressure, profiles is widely thought to result from electrostatic toroidal drifttype instabilities (ion-temperature-gradient (ITG) and trapped-electron modes). These modes will generally be most unstable, in the absence of rotation effects, in the radial regions where the density and temperature gradients for the different plasma species, and thus the total pressure gradient, are the largest. Recently, sheared $\mathbf{E} \times \mathbf{B}$ velocity has been investigated as a mechanism for stabilizing these modes and thus reducing or eliminating the anomalous transport, in localized radial regions of tokamak discharges. If this effect is to explain the experimental observations for ITBs, the stabilization must take place in the region where the radial pressure gradient, and thus the "free energy" available to drive the modes, is the largest. This is possible because the $\mathbf{E} \times \mathbf{B}$ rotation velocity is partially generated by the radial pressure gradient. In particular, in steady state, the radial electric field $E_{r}$ is determined by the radial force-balance relation $E_{r}=V_{I \phi} B_{\theta}-V_{I \theta} B_{\phi}+\left(1 / e_{I} n_{I}\right) d p_{I} / d r$, where $V_{I \phi}$ and $V_{I \theta}$ are the toroidal and poloidal components of an impurity species flow velocity, $B_{\phi}$ and $B_{\theta}$ are the corresponding magnetic field components, and $e_{I}, n_{I}$, and $p_{I}$ are the impurity species charge, number density, and pressure, respectively. If the pressure gradient is large enough in the steep pressure gradient region, it can thus generate a significant radial electric field with a corresponding radial electric field gradient. In favorable circumstances, the flow velocity components can also contribute to the radial electric field. If the field and its gradient are large enough, $\mathbf{E} \times \mathbf{B}$ rotation stabilization of the instabilities can cause a local reduction in anomalous transport in this steep gradient region, allowing the steep pressure gradient to persist. Thus, we can have a situation with a kind of "feed-back loop" in which the steep pressure gradient along with the plasma flows generates an electric field which reduces anomalous transport locally, and thus allows the steep pressure gradient to persist.

In the present work, we will examine some parts of this "loop". In particular, we will calculate the linear growth rates of electrostatic toroidal drift modes without and with sheared $\mathbf{E} \times \mathbf{B}$ rotation effects, and see where the regions of linear stability are with respect to the steep pressure gradient region for various JT-60U ITB and non-ITB cases. If the results are to be consistent with the forgoing argument, we should see stability or near stability in the steep 
gradient region, but possible instability outside that region, for cases with fully-developed ITBs when rotation effects are included. Conversely, when the ITB is degraded or absent, or when rotation effects are not included in the linear growth rate calculation, we should see the region of linear instability spread into the steep gradient region.

For this purpose, we will make use of the comprehensive kinetic microinstability code called the FULL code[1-3]. The FULL code employs the lowest-order ballooning representation[4] and includes all of the kinetic effects thought to be important for toroidal drift modes, including trapped particles, finite Larmor radius effects, banana orbital dynamics, transit and bounce and magnetic drift frequency resonances, and collisions, for all included species. We will also employ the rotation model described in general in Ref. [5] and implemented in the FULL code as described in Ref. [6], along with the one-dimensional, ballooning representation model described in Refs. [7] and [8] of the two-dimensional rotational "eigenfunction shearing" effect described in Ref. [9]. This combined rotation model is qualitatively consistent with the well-known heuristic condition[10] for stabilization of drift modes, in which the mode is expected to be completely stabilized when the $\mathbf{E} \times \mathbf{B}$ shearing frequency, calculated for instance according to the definition in Ref. [11], is comparable to the linear growth rate calculated without rotation. The present model has the advantage, however, that it can be used to calculate the growth rate reduction effects of rotation when the amount of $\mathbf{E} \times \mathbf{B}$ rotation is not large enough to completely stabilize the mode.

We will make use of several cases for the JT-60U tokamak, which is described in general terms in Ref. [12]. Three of these cases correspond to three discharges of varying magnetic shear, one with normal shear, one with weakly reversed shear, and one with strongly reversed shear, only the last of which has an ITB. The other cases correspond to two or three different times in each of three different discharges involving some kind of confinement transition, with only some times corresponding to fully-developed ITBs. For each of these cases, all of the quantities which enter the formula given above for $E_{r}$ are measured experimentally, except for $V_{I \theta}$, for which a neoclassical approximation is employed. The resulting radial profile for $E_{r}$, along with experimentally-derived radial profiles for the densities and temperatures of the included plasma species, are employed in the FULL code linear microinstability calculation. These species are electrons, background deuterium (and/or hydrogen) ions, carbon impurity ions, and hot deuterium (or hydrogen) beam ions. All of these species are used with a Maxwellian equilibrium distribution function, except for the hot beam ions, for which a slowing-down distribution is employed. The MHD equilibrium is computed numerically using the experimentally-derived toroidal current and total pressure profiles, and the plasma boundary shape. 


\section{G. Rewoldt, et al.}

\section{Results}

The first three cases correspond to JT-60U discharges E29826 with normal magnetic shear, E29390 with weakly reversed magnetic shear, and E32423 with strongly reversed magnetic shear[13, 14]. All of these discharges have deuterium background ions and deuterium hot beam ions, along with the carbon impurity and electron species. The total pressure profiles at a chosen time in each discharge are shown in Fig. 1(a), with the normal shear case in red, the weakly reversed shear case in blue, and the strongly reversed shear case in green. The normal shear and weakly reversed shear cases do not display the narrow, steep pressure profile region that characterizes this class of ITBs, while only the strongly reversed shear case pressure profile displays a clear ITB. The linear growth rate curves in Fig. 1(b) including the effects of rotation for the three cases are shown in the corresponding colors. The inner and outer marginally stable radii (or more precisely the radii of the last unstable point calculated before stability) including rotation are marked along the pressure profile curves with solid triangles of the matching colors. The radii of the maximum linear growth rate including rotation for each case are marked with a solid circle along the pressure profile curves in the matching colors also. The inner and outer marginally stable radii without rotation, corresponding to the linear growth rate curves without roation shown in Fig. 1(c), are marked with vertical solid bars along the pressure profile curves in the matching colors also. The linearly unstable region with or without rotation extends far in for the normal shear and weakly reversed shear cases, covering most of the region of pressure rise. Without rotation, only for the case with normal shear, the unstable region extends to the plasma edge region, but is stabilized there when rotation is included. On the other hand, for the strongly reversed shear case with rotation, the unstable region is very narrow, covering only the outermost part of the steep gradient region and the innermost part of the outer relatively flatter pressure gradient region, beyond the footpoint. The footpoint for an ITB is defined as the radial boundary point between the steep pressure gradient region and the outer relatively flatter pressure region. However, if rotation effects are omitted for this case, the inner marginal point moves to be inside the steep pressure gradient region, again most of the way up the pressure rise.

In Fig. 1(d) are shown the corresponding results for the real frequency, $\omega_{r}$ for these discharges. In particular, for the case with strongly reversed shear and with the ITB, the real frequency without rotation is in the electron diamagnetic direction at the smallest unstable radii and in the ion diamagnetic direction at the largest unstable radii. This seems to be a general feature of this class of discharges, sometimes with some additional changes of sign of the real frequency in between. This is due to the fact that the electron density gradient peaks radially inside the ion temperature gradient. With rotation, the real frequency is strongly doppler-shifted for both 
reversed shear cases. In Fig. 1(e) the radial profiles of the safety factor $q$ are shown. For the strongly reversed shear case with the ITB, the unstable region and the ITB region are inside the minimum $q$ radius. Finally, in Fig. 1(f) the corresponding radial electric field profiles are shown. In the present $\mathbf{E} \times \mathbf{B}$ rotation model, $E_{r}$ and $E_{r}^{2}$ and $E_{r}{ }^{\prime}$ can all contribute to rotational stabilization. The strongly reversed shear case with the ITB has a region where $\left|E_{r}^{\prime}\right|$ is large covering the entire steep pressure gradient region.

Figure 2 shows two times in discharge E29728, which also has deuterium background ions and deuterium hot beam ions along with the carbon impurity and electron species, and is marked as for Fig. 1. The earlier time, $t=6.0 \mathrm{~s}$ (in red), has a somewhat weaker ITB, while the later time, $t=6.5 \mathrm{~s}$ (in blue), has a strong ITB, judging by the respective pressure profiles. The plasma has reversed magnetic shear at both of these times. At $t=6.0 \mathrm{~s}$, with the weaker ITB, the unstable region with rotation extends about halfway up the pressure rise, while without rotation it extends to the inside of the steep pressure gradient region. At $t=6.5 \mathrm{~s}$, with the stronger ITB, the unstable region with rotation included is again very narrow and extends only over the outermost part of the steep gradient region and goes only slightly past the footpoint, while with rotation omitted the unstable region extends halfway up the pressure rise.

Figure 3 shows two times in discharge E32844, which has mainly hydrogen background ions with only a small amount of deuterium background ions, and has only hydrogen hot beam ions, again along with the carbon impurity and electron species. The figure is again marked as for Figs. 1 and 2. The earlier time, $t=4.9 \mathrm{~s}$ (in red), is clearly before the confinement transition time and does not have an ITB at all, but instead has a well spread-out pressure gradient. The later time, $t=6.2 \mathrm{~s}$ (in blue), has a somewhat well-developed ITB, with a fairly narrow high pressure gradient region. The plasma has reversed magnetic shear at both of these times. At $t=4.9 \mathrm{~s}$, the linearly unstable region either with or without rotation again covers most of the pressure rise. At $t=6.2 \mathrm{~s}$, on the other hand, the unstable region with rotation in moderately narrow, extending from about one third of the way up the pressure rise in the steep gradient region to a point moderately past the footpoint. For this time with rotation omitted, the linearly unstable region is wider in both directions. For this discharge, density fluctuations have been measured experimentally by means of reflectometry[15, 16]. These measurements show not so much a decrease in the fluctuation amplitude with improved confinement as a large decrease in the radial correlation length. This is consistent with the picture from the linear growth rate calculation here where the toroidal drift mode is not completely stabilized everywhere, but rather the linear eigenfunction is "torn up" by the sheared rotation.

Figure 4 shows three times in discharge E36486, which again has only deuterium background ions and deuterium hot beam ions, along with the carbon impurity and electron species. This discharge has been analyzed in detail from an experimental point of view in Ref. [17]. The 


\section{G. Rewoldt, et al.}

first time, $t=5.85 \mathrm{~s}$ (in red), is at the end of a period of balanced beam injection and has a well developed ITB. The discharge is then switched to dominantly coinjection, and at the second time, $t=6.4 \mathrm{~s}$ (in blue), the ITB has been substantially degraded. The discharge is then switched for a short period to dominantly counterinjection, and then to balanced injection, and the ITB gradually recovers. The third time, $t=6.8 \mathrm{~s}$ (in green), has a partially recovered ITB where the maximum pressure gradient is larger than that at $t=6.4 \mathrm{~s}$ but is not yet as large as that at $t=5.85 \mathrm{~s}$. This evolution process for the time history of the ion temperature gradient at the ITB and for the experimental profiles is shown in Figs. 6 and 7 of Ref. [17]. The plasma has reversed magnetic shear at all three of these times. In the results here in Fig. 4 , for $t=5.85$ $\mathrm{s}$ with the well-developed ITB, the unstable region with rotation included is very narrow and is entirely outside the footpoint, while with rotation omitted the linearly unstable region is much broader and covers the entire steep pressure gradient region. At the second time, $t=6.4 \mathrm{~s}$ with the substantially degraded ITB, the linearly unstable region either with or without rotation covers essentially the entire steep pressure gradient region. At the third time, $t=6.8 \mathrm{~s}$ with the partially recovered ITB, the linearly unstable region with rotation included covers the outer half of the steep gradient region, while if rotation is omitted it covers again essentially the entire steep pressure gradient region.

\section{Conclusions}

The point of all of these comparisons is to look for a particular kind of consistency, as described in Sec. 1. If we assume that the anomalous transport is large only in the linearly unstable radial region, then we need it to be large in regions where the pressure profile is relatively flatter (i.e., beyond the footpoint), and relatively smaller in regions where the pressure gradient is large (i.e., inside the footpoint), in order for the ITB pressure profile to be able to persist. We seem to have this condition for all of the cases in these groups where the ITB is fully developed, along with partial exceptions to this condition when the ITB is partially developed, and complete exceptions to this condition when there is no ITB. Thus, the results seem to be reasonably consistent in this sense for all of these groups of cases. If rotation is turned off in the calculations, the linearly unstable region in several cases extends further inward and spoils this pattern. This is because the stabilizing effect of the $E_{r}$ rotation generated by the large pressure gradient there and by the flows is omitted.

In the innermost and outermost regions, where the pressure profile is relatively flatter, but there is no instability for these toroidal drift modes, we would have to rely on neoclassical transport or on anomalous transport from some instability other than the toroidal drift mode the explain the profile evolution. However, the main point of the present paper is the stabilization 
of toroidal drift modes by sheared $\mathbf{E} \times \mathbf{B}$ rotation in the steep pressure gradient region. No particular allowance has been made here for a decrease in the amount of anomalous transport as the radial marginal points are approached, but this would go along with estimating the anomalous transport by a $\gamma / k_{\perp}^{2}$ type mixing-length formula. This present instability calculation is a strictly linear calculation, and does not allow for a nonlinear upshift in the marginal gradients (as has been seen, for instance, in Ref. [18]), nor for any nonlinear broadening of the anomalous transport region relative to the linearly unstable region (as has been seen, for instance, in Ref. [19]). To some extent, these two nonlinear effects would balance each other out.

In future work, it would be useful to examine other classes of enhanced confinement discharges by the same methods. In particular, the JT-60U experiment and other experiments have several different kinds of ITB discharges, and it would be interesting to see if the same kind of consistency is observed for other kinds as well.

\section{Acknowledgements}

The authors would like to thank Drs. Y. Koide, Y. Kamada, A. Isayama, T. Hatae, and O. Naito for discussions of experimental profiles. This work was supported by United States Department of Energy Contract No. DE-AC02-76-CHO-3073.

\section{References}

[1] Rewoldt, G., Tang, W.M., Chance, M.S., Phys. Fluids 25 (1982) 480.

[2] Rewoldt, G., Tang, W.M., Hastie, R.J., Phys. Fluids 30 (1987) 807.

[3] Kotschenreuther, M., Rewoldt, G., Tang, W.M., Comp. Phys. Comm. 88 (1995) 128.

[4] Frieman, E.A., Rewoldt, G., Tang, W.M., Glasser, A.H., Phys. Fluids 23 (1980) 1750.

[5] Hahm, T.S., Phys. Plasmas 3 (1996) 4658.

[6] Rewoldt, G., et al., Phys. Plasmas 5 (1998) 1815.

[7] Rewoldt, G., Lao, L.L., Tang, W.M., Phys. Plasmas 4 (1997) 3293.

[8] Rewoldt, G., Chance, M.S., Hahm. T.S., Tang, W.M., in Proceedings of the 1998 International Conference on Plasma Physics combined with the 25th European Physical Society Conference on Controlled Fusion and Plasma Physics, Praha, June 29 - July 3, 1998, Edited by Pavlo, P., Europhysics Conference Abstracts, Vol. 22C, published on CD-ROM, paper G046PR (P1.195).

[9] Artun, M., Tang, W.M., Rewoldt, G., Phys. Plasmas 2 (1995) 3384.

[10] Waltz, R.E., Kerbel, G.D., Milovich, J., Phys. Plasmas 1 (1994) 2229.

[11] Hahm, T.S., Burrell, K.H., Phys. Plasmas 2 (1995) 1648. 
[12] Ishida, S., JT-60 Team, Nucl. Fusion 39 (1999) 1211.

[13] Shirai, H., et al., Nucl. Fusion 39 (1999) 1713.

[14] Shirai, H., JT-60 Team, Phys. Plasmas 5 (1998) 1712.

[15] Nazikian, R., et al., in Proceedings of the 17th International Conference on Plasma Physics and Controlled Nuclear Fusion Research, (Yokohama, Japan, October 1998), (International Atomic Energy Agency, Vienna, Austria, 1999), paper IAEA-CN-69/PDP/03, 4 pp, Vol. 4, p. 1689. This paper is available on the web in PDF format at: http://www.iaea.org/programmes/ripc/physics/html/node344.htm.

[16] Shinohara, K., et at., Proceedings of the 26th European Physical Society Conference on Plasma Physics and Controlled Fusion, Maastricht, 1999, (EPS, 1999).

[17] Sakamoto, Y., et al., "Characteristics of Internal Transport Barrier in JT-60U Reversed Shear Plasmas", in Proceedings of the 18th International Conference on Plasma Physics and Controlled Nuclear Fusion Research, (Sorrento, Italy, October 2000), (International Atomic Energy Agency, Vienna, Austria, 2001), paper IAEA-CN-77/EX6/4.

[18] Dimits, A.M., et al., Phys. Plasmas 7 (2000) 969.

[19] Mynick, H.E., Parker, S.E., Bull. Am. Phys. Soc. 41 (1996) 1359. 

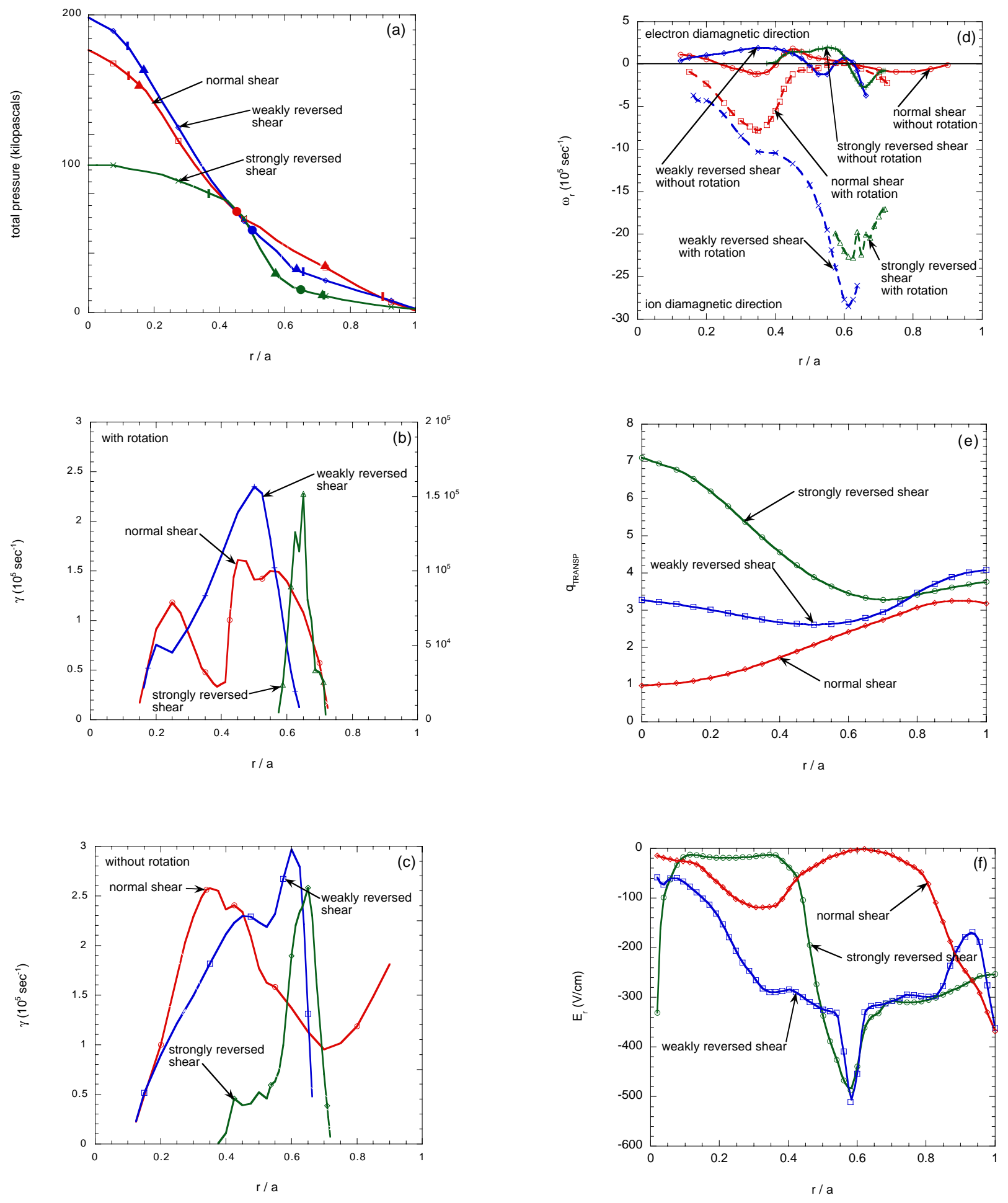

Figure 1. (a) Total pressure, linear growth rate (b) with rotation and (c) without rotation, (d) real frequency, $(e) q$, $(f) E_{r}$ versus r/a for JT-60U discharges E29826 (in red), E29390 (in blue), and E32423 (in green). 

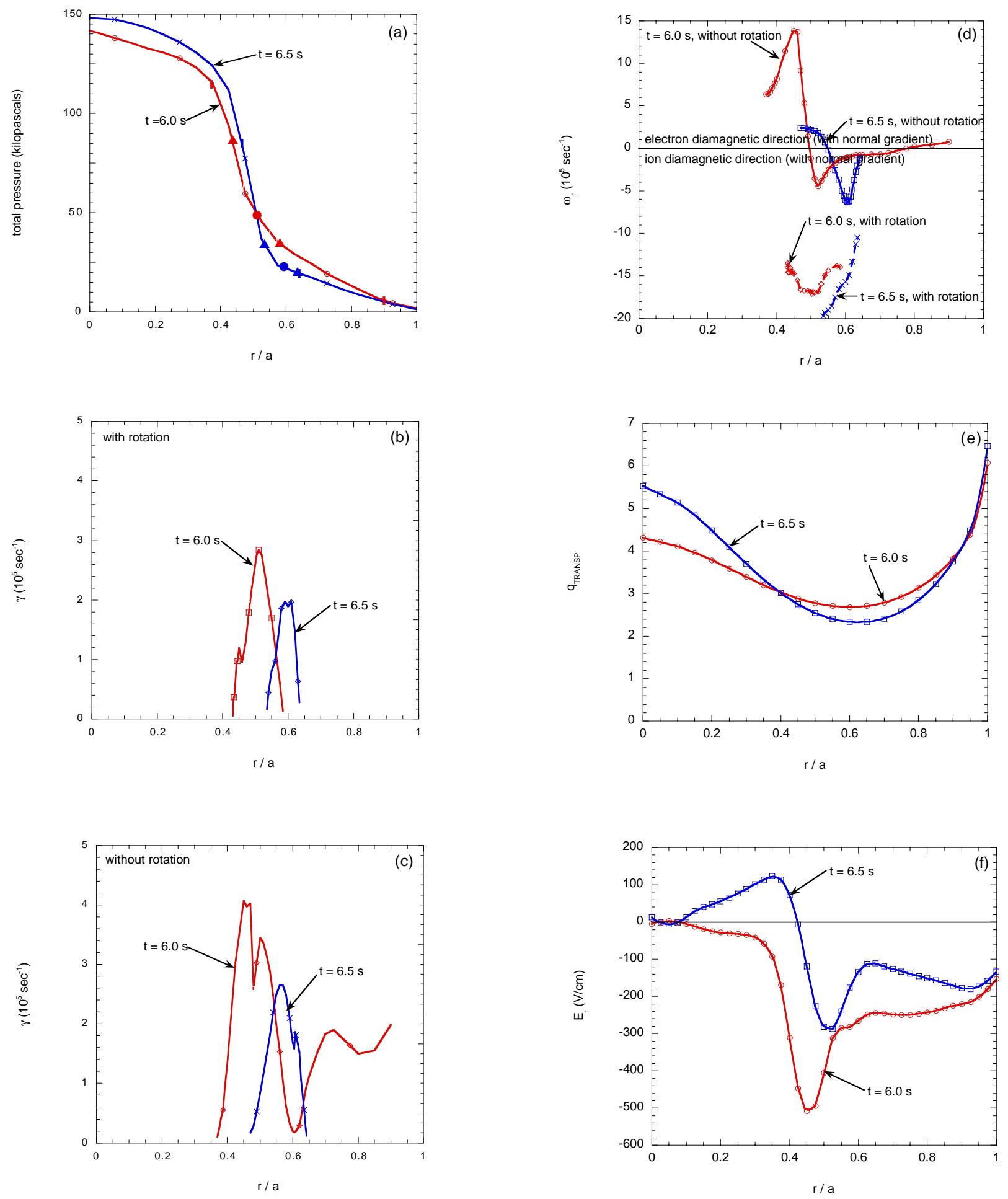

Figure 2. (a) Total pressure, linear growth rate (b) with rotation and (c) without rotation, (d) real frequency, $(e) q$, and $(f) E_{r}$ versus r/a for JT-60U discharge E29728 at $t=6.0 \mathrm{~s}$ (in red) and $t=6.5 \mathrm{~s}$ (in blue). 

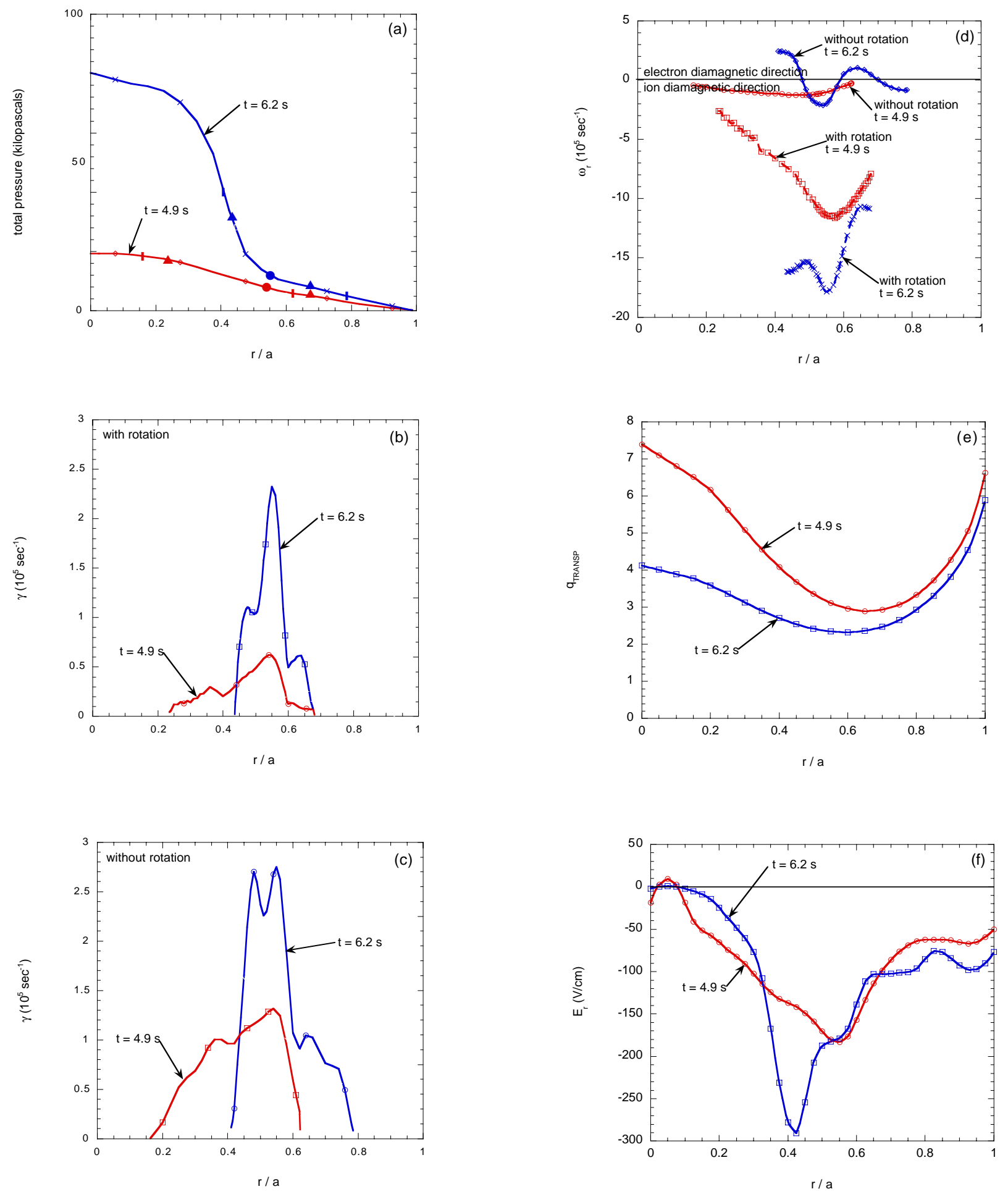

Figure 3. (a) Total pressure, linear growth rate (b) with rotation and (c) without rotation, $(d)$ real frequency, $(e) q$, and $(f) E_{r}$ versus r/a for JT-60U discharge E32844 at $t=4.9 \mathrm{~s}$ (in red) and $t=6.2 \mathrm{~s}$ (in blue). 

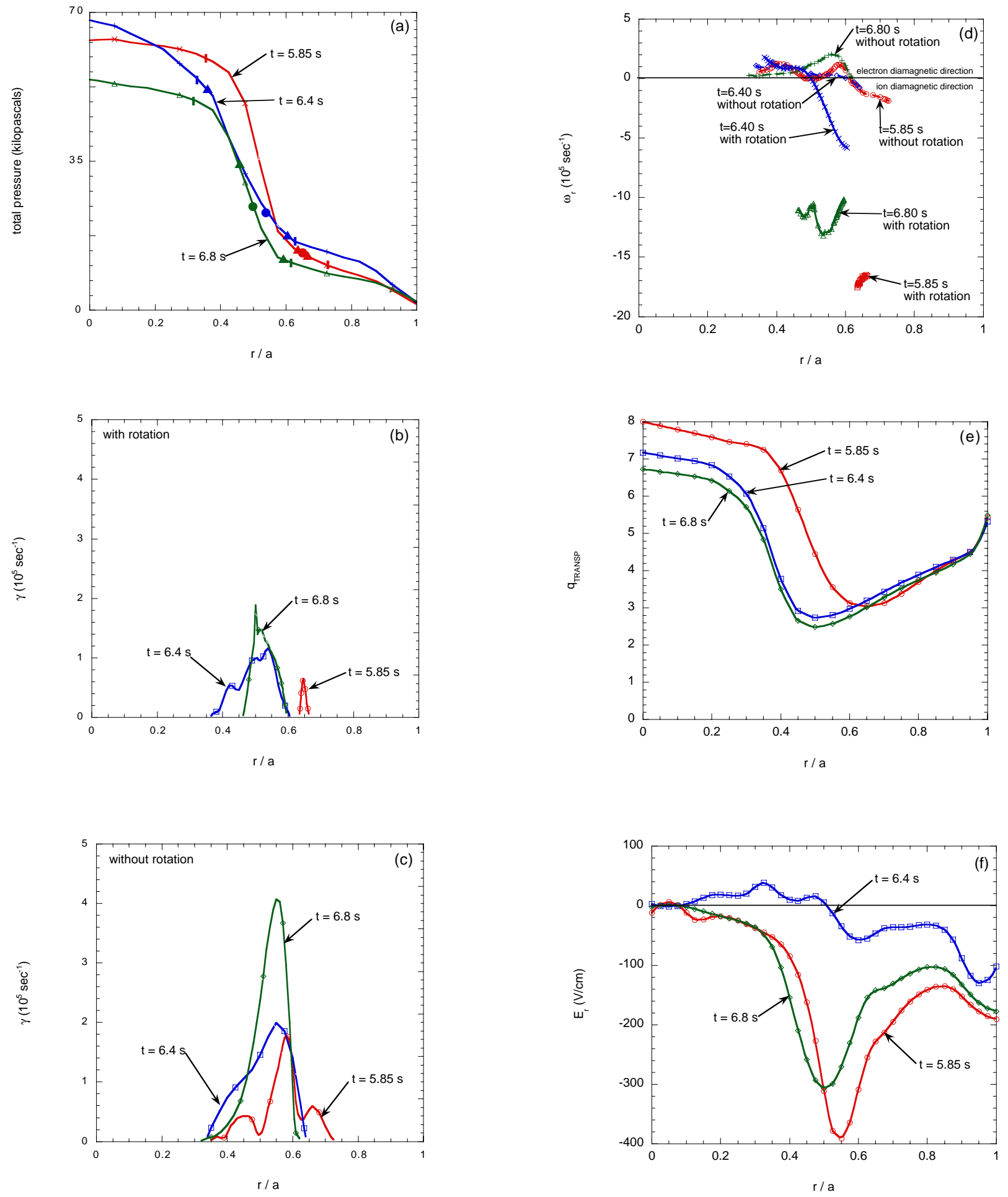

Figure 4. (a) Total pressure, linear growth rate (b) with rotation and (c) without rotation,

(d) real frequency, $(e) q$, and $(f) E_{r}$ versus r/a for JT-60U discharge E36486 at $t=5.85 \mathrm{~s}$ (in red), $t=6.4 \mathrm{~s}($ in blue $)$, and $t=6.8 \mathrm{~s}($ in green $)$. 


\section{External Distribution}

Plasma Research Laboratory, Australian National University, Australia

Professor I.R. J ones, Flinders University, Australia

Professor J oão Canalle, Instituto de Fisica DEQ/IF - UERJ , Brazil

Mr. Gerson O. Ludwig, Instituto Nacional de Pesquisas, Brazil

Dr. P.H. Sakanaka, Instituto Fisica, Brazil

The Librarian, Culham Laboratory, England

Library, R61, Rutherford Appleton Laboratory, England

Mrs. S.A. Hutchinson, JET Library, England

Professor M.N. Bussac, Ecole Polytechnique, France

Librarian, Max-Planck-Institut für Plasmaphysik, Germany

J olan Moldvai, Reports Library, MTA KFKI-ATKI, Hungary

Dr. P. Kaw, Institute for Plasma Research, India

Ms. P.J . Pathak, Librarian, Insitute for Plasma Research, India

Ms. Clelia De Palo, Associazione EURATOM-ENEA, I taly

Dr. G. Grosso, Instituto di Fisica del Plasma, Italy

Librarian, Naka Fusion Research Establishment, J AERI, J apan

Library, Plasma Physics Laboratory, Kyoto University, J apan

Research Information Center, National Institute for Fusion Science, J apan

Dr. O. Mitarai, Kyushu Tokai University, J apan

Library, Academia Sinica, Institute of Plasma Physics, People's Republic of China

Shih-Tung Tsai, Institute of Physics, Chinese Academy of Sciences, People's Republic of China

Dr. S. Mirnov, Triniti, Troitsk, Russian Federation, Russia

Dr. V.S. Strelkov, Kurchatov Institute, Russian Federation, Russia

Professor Peter Lukac, Katedra Fyziky Plazmy MFF UK, Mlynska dolina F-2, Komenskeho Univerzita, SK-842 15 Bratislava, Slovakia

Dr. G.S. Lee, Korea Basic Science Institute, South Korea

Mr. Dennis Bruggink, Fusion Library, University of Wisconsin, USA

Institute for Plasma Research, University of Maryland, USA

Librarian, Fusion Energy Division, Oak Ridge National Laboratory, USA

Librarian, Institute of Fusion Studies, University of Texas, USA

Librarian, Magnetic Fusion Program, Lawrence Livermore National Laboratory, USA

Library, General Atomics, USA

Plasma Physics Group, Fusion Energy Research Program, University of California at San Diego, USA

Plasma Physics Library, Columbia University, USA

Alkesh Punjabi, Center for Fusion Research and Training, Hampton University, USA

Dr. W.M. Stacey, Fusion Research Center, Georgia Institute of Technology, USA

Dr. J ohn Willis, U.S. Department of Energy, Office of Fusion Energy Sciences, USA

Mr. Paul H. Wright, Indianapolis, Indiana, USA 
The Princeton Plasma Physics Laboratory is operated by Princeton University under contract with the U.S. Department of Energy.

\author{
Information Services \\ Princeton Plasma Physics Laboratory \\ P.O. Box 451 \\ Princeton, NJ 08543
}

Phone: 609-243-2750

Fax: 609-243-2751

e-mail: pppl_info@pppl.gov

Internet Address: http://www.pppl.gov 